Article

\title{
High-Performance Pressure Sensor for Monitoring Mechanical Vibration and Air Pressure
}

\author{
Yancheng Meng ${ }^{1,2}$, Hongwei $\mathrm{Li}^{2}$, Kunjie $\mathrm{Wu}^{2}$, Suna Zhang ${ }^{2}$ and Liqiang $\mathrm{Li}^{1,2, *}$ \\ 1 School of Nano-Technology and Nano-Bionics, University of Science and Technology of China, \\ Hefei 230026, China; ycmeng2015@sinano.ac.cn \\ 2 Suzhou Institute of Nano-Tech and Nano-Bionics (SINANO), Chinese Academy of Sciences, \\ Suzhou 215123, China; hwli2015@sinano.ac.cn (H.L.); kjwu2014@sinano.ac.cn (K.W.); \\ snzhang2014@sinano.ac.cn (S.Z.) \\ * Correspondence: lqli2014@sinano.ac.cn; Tel.: +86-512-6287-2829
}

Received: 4 April 2018; Accepted: 25 May 2018; Published: 27 May 2018

\begin{abstract}
To realize the practical applications of flexible pressure sensors, the high performance (sensitivity and response time) as well as more functionalities are highly desired. In this work, we fabricated a piezoresistive pressure sensor based on the micro-structured composites films of multi-walled carbon nanotubes (MWCNTs) and poly (dimethylsiloxane) (PDMS). In addition, we establish efficient strategies to improve key performance of our pressure sensor. Its sensitivity is improved up to $474.13 \mathrm{kPa}^{-1}$ by minimizing pressure independent resistance of sensor, and response time is shorten as small as $2 \mu$ s by enhancing the elastic modulus of polymer elastomer. Benefiting from the high performance, the functionalities of sensors are successfully extended to the accurate detection of high frequency mechanical vibration $(\sim 300 \mathrm{~Hz})$ and large range of air pressure (6-101 kPa), both of which are not achieved before.
\end{abstract}

Keywords: pressure sensor; ultra-fast response; mechanical vibration and air pressure

\section{Introduction}

Pressure sensor is one of key components of flexible and wearable electronic products [1-14]. For realizing practical applications, the high performance (especially the high sensitivity and fast response time) and diverse functionalities (i.e., capacity for detecting more kinds of stimulus) are highly desired $[15,16]$. However, the sensitivities of current pressure sensors are generally in the range from several to one hundred of $\mathrm{kPa}^{-1}[15,17-21]$, and the response times are at the level of milliseconds or larger $[16,20,22-25]$. Specially, the piezoresistive pressure sensor, which is operated via changing the contact area and thus the resistance to tune the output current, have great potential in flexible systems, while their sensitivity are still in very low levels $[10,12,25]$. For example, Choong et al. reported a classical piezoresistive pressure sensor that is based on the conductive polymer coated cone-shape geometry, in which the sensitivity is only $10.32 \mathrm{kPa}^{-1}$. Until now, no efficient strategy is developed to greatly improve the performance of piezoresistive pressure sensor far beyond the current level. In addition, regarding the functionalities (application fields) of pressure sensors, most of them aimed to monitor or reflect the mechanical state of human body or robot [16,26-31]. While there are rare applications in detecting the mechanical quantity in the environment such as high frequency (over hundreds of $\mathrm{Hz}$ ) mechanical vibration and air pressure, both of which are closely related to human life. Epidemiological and experimental evidences suggest that if human is exposed to vibration at frequencies higher than $100 \mathrm{~Hz}$, the risk of injury dramatically increases [32]. Conventionally, the microelectromechanical systems (MEMS) were proposed to detect the vibrations, while these devices are intrinsically rigid, which are unfavorable to realize flexible 
electronics applications. Recently, numerous flexible sensors such as pressure sensor and strain sensor were proposed to detect the classical mechanical quantities [22-25]. However, they have very limited performance to detect vibrations, especially in high frequency region. To realize the detection of high frequency vibration, fast response should be achieved which is difficult because the polymer elastomers employed in these sensors have a poor response to high frequency excitations for their viscoelasticity and poor mechanical recovery.

Air pressure is also an important environmental factor in the daily life, especially for someone exposed to positive or negative pressure constantly. The traditional air pressure gauge is rigid and cumbersome. Optical fiber-based air pressure sensors were fabricated with compact size in recent years $[33,34]$, while a complex test system is needed for signal detection which may prevent these devices from being used in flexible electronics at present. Therefore, it is a necessary to propose another light, flexible, wearable, easily detectable air pressure sensor.

According to the working principle, piezoresistive pressure sensor has potential for realizing the detection of high frequency vibration and air pressure. Meanwhile, the air pressure gauge based on flexible pressure sensor is not reported until now. The limited functionalities might stem from the relatively low performance as well as the lack of proper strategy for the device fabrication.

In this article, we present a simple yet efficient strategies to improve the performance of our sensor to far beyond the current level of the piezoresistive pressure sensors. The sensitivity is improved to be a high level of about $474 \mathrm{kPa}^{-1}$ and response/relaxation time is shorten as small as $2 \mu \mathrm{s} / 74 \mu \mathrm{s}$. Meanwhile, the sensors show large range of pressure sensing from $0 \mathrm{~Pa}$ to $110 \mathrm{kPa}$ and a low detection limit of $0.6 \mathrm{~Pa}$. Benefiting from these outstanding properties, we prepare vibration sensor and air presser sensor based on our pressure sensor, which can be used to detect the vibration in the frequency range of 1 to $\sim 300 \mathrm{~Hz}$ as well as air pressure from 6 to $110 \mathrm{kPa}$.

\section{Materials and Methods}

\subsection{Material Synthesis}

Trichloromethane was used to dissolve the poly (dimethylsiloxane) (PDMS) and allowed dispersion of multi-walled carbon nanotubes (MWCNTs). $2 \mathrm{wt} \%$ was chosen as the quality ratio of MWCNTs (purchased from Shichuang Company, Changzhou, China; outer diameter: 15-20 nm) to PDMS for all of our samples. To start mixing, the PDMS base polymer, dry MWCNTs and Trichloromethane were added into beaker successively. Then the beaker was positioned on top of heat source with the temperature $80^{\circ} \mathrm{C}$, and the mixture was continual stirred for $4 \mathrm{~h}$ to promote MWCNTs dispersing into PDMS and trichloromethane evaporating from the mixture. After trichloromethane reached full evaporation, the PDMS curing agent (1:10 weight ratio to base polymer) was added into the mixture and stirred for $5 \mathrm{~min}$. Then the conductive materials were readied and waited to spin-coating as thin layer.

\subsection{Micro-Structured Composite Films Fabrication}

Silicon slice was chosen as mold which have recessed similar-pyramid structures, which was acquired from Shichuang Company, Changzhou, China. The surface of the silicon mold was coated with OTS (octadecyltrichlorosilane) to avoid the adhesion of the composite film. Then used the spin-coating method to prepare the micro-structured composite films, after degassing and curing in the vacuum chamber with $100{ }^{\circ} \mathrm{C}$ temperature and $100 \mathrm{~Pa}$ vacuum level, the micro-structured composite layers were peeled off.

\subsection{Signal Detection System}

Keithley 4200 was used to apply the voltage into the sensor. When constant voltage is applied to the sensor, the current will flow through the apex of pyramid-like microstructure to the indium tin oxide poly (ethyleneterephthalate) (ITO-PET) layer. The sensor is operated via changing the 
contact area and thus the resistance under varying pressures. We successively applied a series of step-like pressures to the sensor, and then released the pressure in reverse sequence. At the same time, the output current can be recorded by the Keithley 4200 in situ under various pressure condition.

\subsection{Vibrations Calibration}

The commercial standard vibration sensor should be fixed on the mechanical vibration source. It can monitor the vibration in situ. When the mechanical vibration source under operating, vibration's accelerations and frequencies can be measured by standard sensor and recorded in computer.

\subsection{Air Pressure Sensor Fabrication}

For the first step, the high-purity paraffin was prepared as adhesive. Second, we put the ITO-PET layer on oven and wait the temperature upper to $90{ }^{\circ} \mathrm{C}$, then paint high-purity paraffin on the ITO-PET layer with a closed line frame and put the indium tin oxide (ITO)-coated flexible poly (ethyleneterephthalate) (PET) layer off oven, waiting it cool to room temperature. The third step is that the conductive layer was stacked onto ITO-PET layer matched the line frame of high-purity paraffin and heat the device for the temperature upper to $75^{\circ} \mathrm{C}$ under different vacuum degree. Two minutes later turn off the heater and wait the device cooling to room temperature naturally. Finally, ITO-PET layer and conductive layer pasted together along the closed paraffin line frame automatically, and the vacuum level was hold between two layers as same as the vacuum degree in packaging condition.

\section{Results and Discussion}

\subsection{Sensor Fabrication and Working Principle}

Figure 1 schematically illustrated the fabricated process of our sensor. The former conductive layer was prepared by doping MWCNTs into PDMS, which has pyramid-like patterns (Figure 1a), being the replica of that on the recessed pyramid-like silicon mold. MWCNTs were used as filler materials because of their exceptional mechanical, electrical, thermal, and magnetic properties coupled with very large interfacial contact area $[35,36]$. At the same time, the blending of MWCNTs may enhance the elastic modulus of PDMS films. This property may render the micro-structured flexible films to have fast recovery progress and avoid wrinkling under operation in ultra-high pressure range as demonstrated in the contents below.

(a)

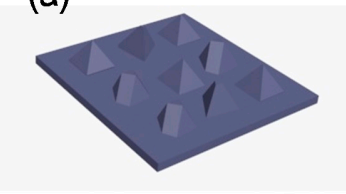

(b)

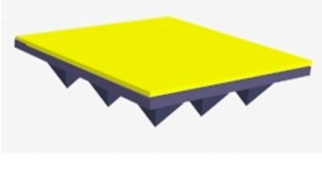

(c)

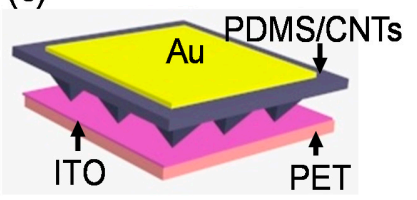

Figure 1. Schematic illustration of sensor fabrication. (a) Conductive layers with micro-structures are cured and peeled off from Si mold; (b) Au was deposited on the reverse side of conductive layers; (c) A sensor unit was constructed through a sandwich structure comprising the micro-structured conductivity layers and indium tin oxide (ITO)-coated poly (ethyleneterephthalate) (PET) film.

$\mathrm{Au}$ was deposited on the reverse side of conductive layers based on the vacuum evaporation coating method (Figure 1b). Subsequently, the micro-structured conductivity layer is affixed to a flat conductive ITO-PET film face-to-face, which has a sandwich structure (Figure 1c).

The sensor is consists of two conductive layers: one is poly (ethyleneterephthalate) (PET) substrate coated with indium tin oxide (ITO), and the other is micro-structured composite films with the uniform thickness of about $100 \mu \mathrm{m}$. It has pyramid-like patterns (Figure 2a,b) on one side replicated from the silicon mold. The largest height of these patterns is approximately uniform, and the value is about 
$4 \mu \mathrm{m}$. On the other side, one high conductive Au film with controlled area was deposited as top conductive electrode.

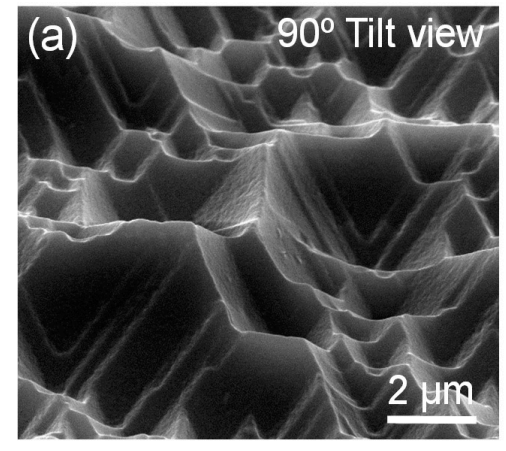

(c)

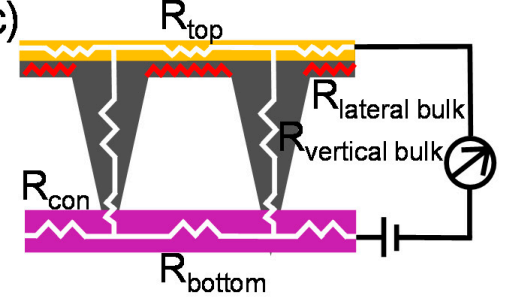

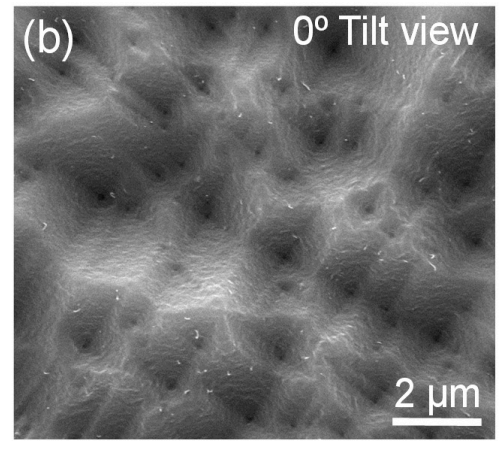

(d)

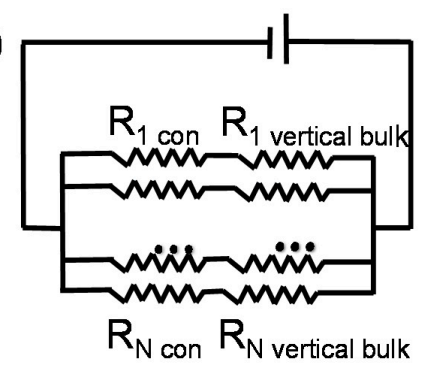

Figure 2. SEM images of conductive layer with pyramid-like patterns (a) with $90^{\circ}$ tilt view and (b) with $0^{\circ}$ tilt view. (c) A simple circuit model corresponding to random individual pyramid-like microstructure. The pressure independent lateral bulk transporting resistance $\left(R_{\text {lateralbulk, }}\right.$, red folding line) is replaced by top electrode resistance $\left(R_{\text {top }}\right)$. (d) The proposed equivalent circuit diagram of entire sensor.

The Au film is highly important for the sensitivity improvement due to the following reasons: The total resistance of pressure sensor mainly consists of contact resistance $\left(R_{\text {con }}\right)$ and bulk resistance $\left(R_{\text {bulk }}\right)$, so it would be favorable to achieve high sensitivity if $R_{\text {con }}$ and $R_{\text {bulk }}$ may change with pressure simultaneously. Generally, $R_{\text {con }}$ strongly depends on the pressure, but $R_{\text {bulk }}$ shows weak or no dependence on the pressure in some pressure sensors with large spacing distance (several $\mathrm{mm}$ to $\mathrm{cm}$ ) between two high conductive electrodes, which might be one of main reasons for the limited sensitivity. To overcome this problem, minimization or elimination of $R_{\text {bulk }}$ would be an effective way. In our work, the lateral bulk transporting resistance $\left(R_{\text {lateralbulk }}\right)$ of the top conductive layer is replaced by the negligible Au electrode resistance $\left(R_{\text {top }}\right)$ as Figure 2c illustrated, which successfully eliminates the lateral bulk resistance that shows weak dependence on pressure. Therefore, the total resistance of individual of pyramid-like microstructure is sum of vertical bulk resistance and contact resistance (Figure 1c), both of which are highly dependent on the pressure as demonstrated and explained in the contents below. At the same time, each pyramid-like microstructure is connected in parallel mode (Figure 2d), so the total resistance of the sensor is highly depended on the pressure too. This fact means the total resistance of the sensor device can be effectively tuned by the changes of pressure, which may enhance the sensitivity significantly. When constant voltage is applied to the sensor by Keithley 4200, the current will flow through the apex of pyramid-like microstructure to the ITO-PET layer. The sensor is operated via changing the contact area and thus the resistance under varying pressures.

\subsection{High Sensitivity and Theoretical Analysis}

The electrical properties of the pressure sensor with Au film area of $4.4 \times 10^{-5} \mathrm{~m}^{2}$ (larger area) were measured under variable loads. From current-voltage curves (Figure 3a), it can be seen that the 
sensor's resistance exhibits an obvious decreases as the applied pressure increases. Under the pressure of $0 \mathrm{~Pa}$, the current reaches to $3.79 \times 10^{-8} \mathrm{~A}$ at the voltage of $1 \mathrm{~V}$, which is set as the initial current.
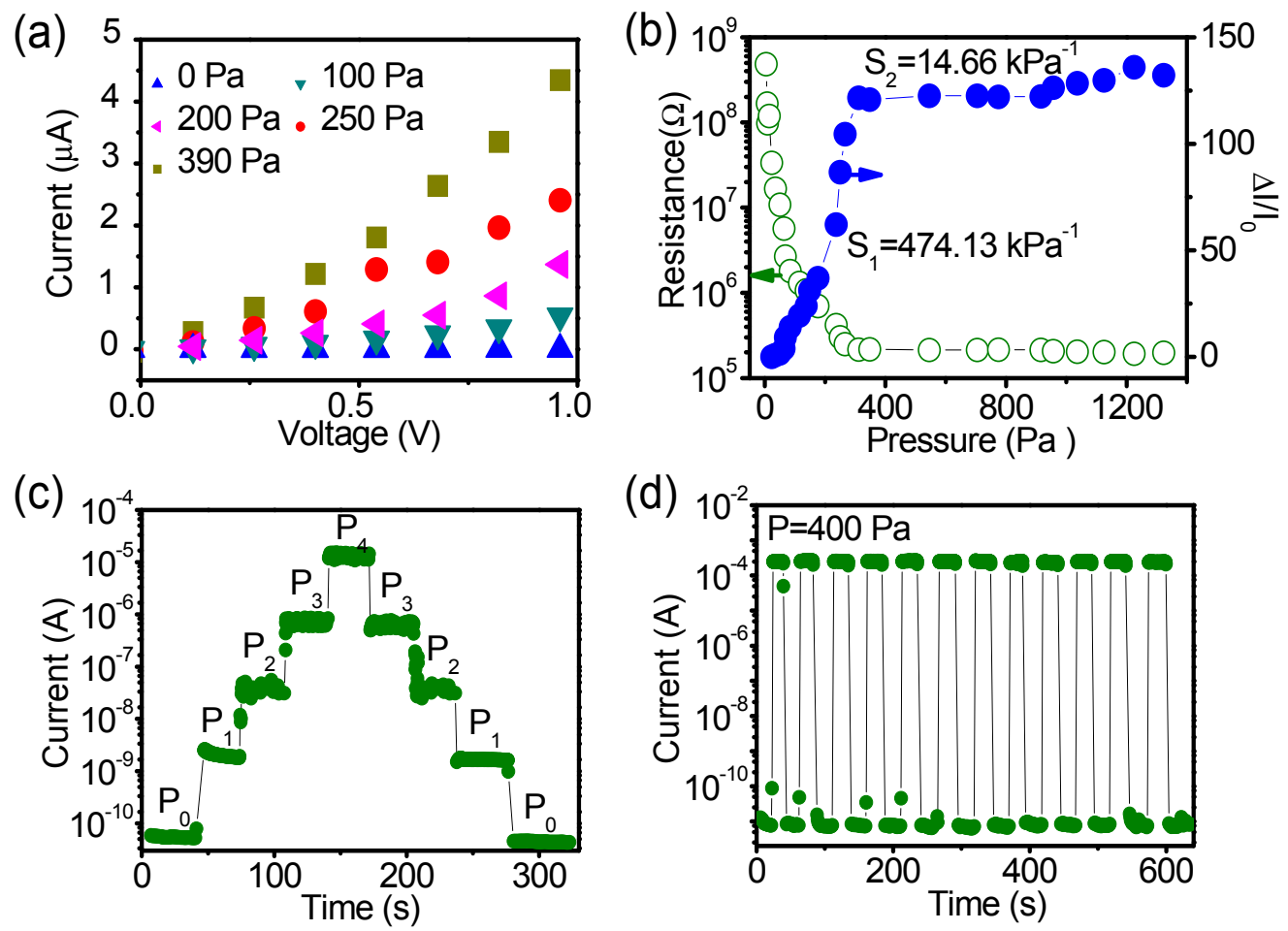

Figure 3. Sensitivity and repeatability of the sensor with large area of Au film $\left(4.4 \times 10^{-5} \mathrm{~m}^{2}\right)$. (a) Current-voltage curves of the device under different amount of pressure loading; (b) The dependency of sensitivity and total resistance on pressure under the same pressure range (0 to $1400 \mathrm{~Pa}$ ); (c) Current response of the sensor under step pressure and certain applied voltage of $5 \mathrm{~V}, P_{0}=0 \mathrm{~Pa}$, $P_{1}=11 \mathrm{~Pa}, P_{2}=25 \mathrm{~Pa}, P_{3}=52 \mathrm{~Pa}$ and $P_{4}=118 \mathrm{~Pa}$; (d) Cycle test of sensor under pressure of $400 \mathrm{~Pa}$ and voltage of $1 \mathrm{~V}$.

Sensitivity is the most important parameter of pressure sensor, because it determines the measurement accuracy and effectiveness of the device [23]. In order to calculate the sensitivity of our sensor, an elaborate measurement was performed for the pressure increasing from 0 to $1400 \mathrm{~Pa}$. The sensors showed maximum resistance without loading. The application of pressure leaded to a sharp decline of the total resistance of the device until an inflection point appeared at $400 \mathrm{~Pa}$, and then this tendency approached saturation with applying higher pressures (Figure 3b). The different dependencies of resistance on pressure enable the curve of relative variation of current $\left(\Delta I / I_{0}\right)$ versus pressure to show distinct tendency with two regions. Near linear region (0 to $\left.400 \mathrm{~Pa}\right)$ shows the sensitivity up to $474.13 \mathrm{kPa}^{-1}$ and approximate saturated region (400 to $1400 \mathrm{~Pa}$ ) shows sensitivity of $14.66 \mathrm{kPa}^{-1}$, respectively, which are plotted as blue curve in Figure $3 \mathrm{~b}$. The sensitivity in low pressure regime is quite high compared with the reported values of piezoresistive pressure sensors calculated with the standard method $[10,12,25]$. Then a similar test was performed in the large pressure range from 5 to $110 \mathrm{kPa}$, and the sensitivity of $10.46 \mathrm{kPa}^{-1}$ was got as shown in Figure S1. These results indicate that our sensors have excellent performance at low pressure region and larger pressure measurement range.

To further testify the high sensitivity and repeatability of our sensor in low pressure regime, we successively applied a series of step-like pressures with $0,11,25,52,118 \mathrm{~Pa}$ to the sensor, and then released the pressure in reverse sequence. The current changed without hysteresis, and retained nearly the same level for the certain pressure during the loading and unloading the pressure (Figure $3 \mathrm{c}$ and Figure S2). Furthermore, the current changed over several orders of magnitude at the applied pressure 
of $118 \mathrm{~Pa}$, confirming the high sensitivity. At same time, another repeatability tests were performed under pressure switching between 0 to $400 \mathrm{~Pa}$ (Figure 3d) and 0 to $14 \mathrm{~Pa}$ (Figure S3), respectively, all of which confirmed the high repeatability.

The above experimental results show that our sensor produces a high sensitivity (about $480 \mathrm{kPa}^{-1}$ ), which is higher than most of reported results. To interpret this performance, a mathematical calculation and analysis are performed. For simplicity purposes, the pyramid-like microstructure was approximated as cone in the process of calculation. Based on Holm's theory and steady electric field approximation $[37,38]$, contact and vertical bulk resistant can be calculated (Supporting Discussion S1, Figure S4), respectively, and the total resistance of sensor can be expressed as Equation (1)

$$
R_{\text {total }}=\frac{1}{N} \cdot R_{\mathrm{n} \text { total }}=\frac{1}{N} \cdot\left(C \cdot \frac{1}{r_{1 \mathrm{n}}}+D \cdot \frac{1}{r_{2 \mathrm{n}}}\right)
$$

$r_{1 \mathrm{n}}$ is radius of cone's section deformed under pressure, $r_{2 n}$ is radius of cone's back surface that is a constant (schematically illustrates at Figure S5). $C$ and $D$ are constants, which are abbreviations of the product of several constants (Supporting Discussion S1). $N$ is number of pyramid-like microstructure below the Au film.

For a certain sensor, $N, C, D$ and $r_{2 n}$ are constants, so $R_{\text {total }}$ is proportional to $1 / r_{1 \mathrm{n}}$. With the increasing of pressure, $1 / r_{1 n}$ will decrease, rendering $R_{\text {total }}$ to reduce continuously. In the other words, the sensor's total resistance is highly dependent on the pressure changes.

Based on Equation (1), we have following relation

$$
\frac{d R_{\text {total }}}{d r_{1 \mathrm{n}}}=-\frac{C}{N} \cdot r_{1 \mathrm{n}}^{-2}
$$

That is, the slope of Equation (1) is $-1 / r^{2}{ }_{1 n}$, and its absolute value decreases with the increasing of $r_{1 \mathrm{n}}$. According to the basic principle of power equation, Equations (1) and (2) indicate that with the increasing of pressure, $R_{\text {total }}$ will decrease continuously with gradually weakened tendency, until approaching to a constant ( $r_{1 \mathrm{n}}$ will approach to $r_{2 \mathrm{n}}$ under enough pressure), which is in good accordance with the experimental result (Figure $3 b$ ).

In fact, the pressure sensor with micro-pyramid structure generally shows nonlinear dependency of resistance or current on the applied pressure, and the sensitivity generally exhibits a reduction in the high-pressure regime $[1,9,16]$. However, until now this phenomenon is still not well-understood in a rational mode. Equations (1) and (2) provide a mathematical and precise explanation for the nonlinear sensing curve for the first time.

Generally, the sensitivity of pressure sensor is defined as the following relation

$$
S=\frac{I-I_{0}}{I_{0} \cdot \Delta P}=\frac{R_{0}}{\Delta P} \cdot \frac{1}{R_{\text {total }}}-\frac{1}{\Delta P}
$$

$I_{0} / R_{0}$ and $I / R_{\text {total }}$ are current/total resistance of the sensor without and with load, respectively. $\Delta P$ is magnitude of pressure changes. Obviously, $S \propto\left(1 / R_{\text {total }}\right)$, indicating the sensor's total resistance is key parameter to optimize its sensitivity.

As Equation (3) shown, the sensor's sensitivity is proportional to $1 / R_{\text {total }}$, and $1 / R_{\text {total }}$ is proportional to the value of $N$ (Equations (1) and (2)). So we can improve the sensor's sensitivity through increasing the value of $N$. Our sensor consists of conductive top layer and bottom layer. The top layer have vertical resistance $\left(R_{\mathrm{h}}\right)$ originating from layer's thickness $(h=100 \mu \mathrm{m})$ and lateral resistance $\left(R_{\Delta L}\right)$ originating from the displacement $(\Delta L)$ in layer, and the sum of them are bulk resistance of top layer (or the sensor). If depositing $\mathrm{Au}$ on the entire opposite side of top layer (Figure 1b), it will have a uniform electric potential under the certain voltage, and $R_{\Delta L}$ can be ignored. This indicates that all of the pyramid-like microstructures below the Au film are effective conductive access schematically illustrated as Figure 4a. If the area of deposited $\mathrm{Au}$ film is smaller than the surface area of top 
layer (Figure $4 \mathrm{~b}$ inset), $R_{\Delta L}$ originated from the region near outside the $\mathrm{Au}$ film cannot be ignored. When the length of displacement $(\Delta L)$ away from Au film edge is much larger than layer's thickness $(100 \mu \mathrm{m}), R_{\Delta L}$ will be much larger than $R_{\mathrm{h}}$ too. It indicates that the corresponding conductive access far away from the Au film edge can be neglected (Figure 4a, right side). So the effective area of electric transmission approximates to the area of $\mathrm{Au}$ film, and the values of $N$ (conductive access) can be approximated to the number of pyramid-like microstructures below the area of Au film. It thus implies that the value of $N$ can be easily modulated by changing the area of Au film.

(a)

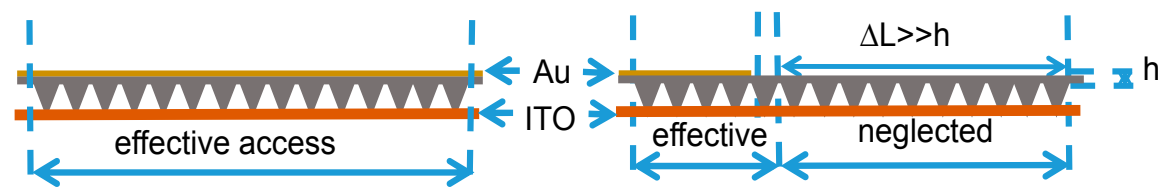

(b)

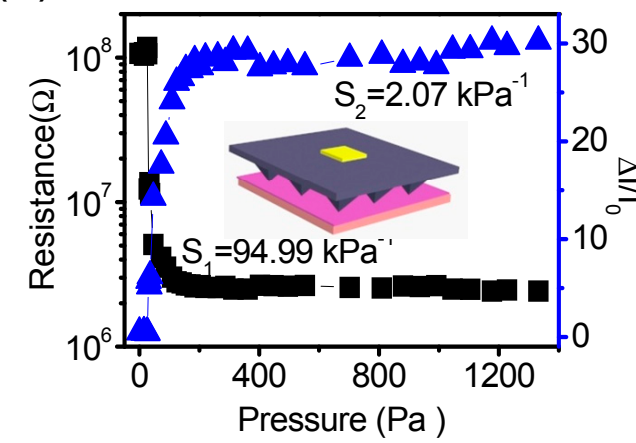

(c)

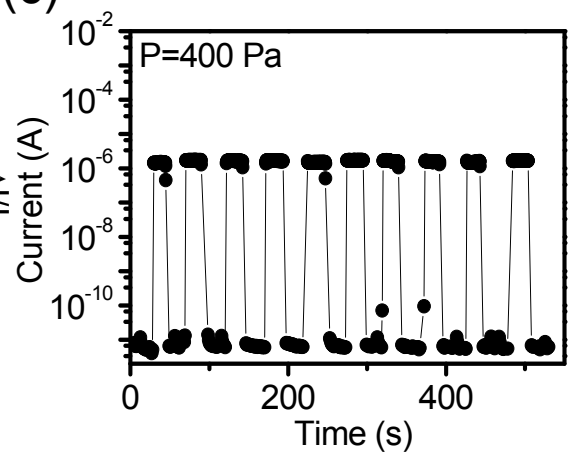

Figure 4. (a) Schematic illustration of the difference of the effective access for the sensor with different areas of $\mathrm{Au}$ film. The performance of sensor with smaller area of Au film $\left(1 \times 10^{-6} \mathrm{~m}^{2}\right)$; $(\mathbf{b})$ the dependency of sensitivity and total resistance on pressure, inset is the schematic illustration of the sensor with smaller area of Au film; (c) relaxation and response curve under pressure switching between 0 and $400 \mathrm{~Pa}$.

Therefor the ratio of total resistance can be expressed as the Equation (4) for two sensors with same size and different $\mathrm{Au}$ film areas.

$$
\frac{R_{\text {total }}^{\prime}}{R_{\text {total }}}=\frac{N}{n} \approx \frac{A}{a}
$$

$A, a$ and $N, n$ are the area of Au films and the number of pyramid-like microstructure below the $\mathrm{Au}$ films for two different sensors, respectively.

Based on Equations (3) and (4), under the same magnitude of pressure change, the ratio of sensitivity can be expressed as Equation (5) for two sensors that with same size and different $\mathrm{Au}$ film areas.

$$
\frac{S}{S^{\prime}} \approx \frac{I_{0}^{\prime} \cdot V}{I_{0} \cdot V^{\prime}} \cdot \frac{A}{a}
$$

It indicates that under the known values of initial current $\left(I_{0}\right)$ and voltage $(V)$, the difference of sensitivity of two sensors is only relate to the area of Au film.

To verify the above analyses, we prepared a similar sensor with a smaller area of Au film $\left(1 \times 10^{-6} \mathrm{~m}^{2}\right.$, Figure $4 \mathrm{~b}$ inset). Its sensitivity decreased obviously to only $94.99 \mathrm{kPa}^{-1}$ at low pressure region (Figure $4 \mathrm{~b}$ ), which is 5 times less than that of sensor with larger area $\left(4.4 \times 10^{-5} \mathrm{~m}^{2}\right)$ of Au film (Figure $3 b$ ). The ratio is close to the theoretical prediction (8.8 times). It demonstrates the reliability of our theoretical model. On the other hand, under the same cycle test process switching pressure 
between 0 and $400 \mathrm{~Pa}$, the current changes of sensor with larger area of $\mathrm{Au}$ film (Figure 3d) is two orders of magnitude larger than that of the sensor with smaller area of Au film (Figure 4c).

According to the above experimental results and theoretical analyses, one strategy for improving sensitivity can be deduced as follow: the lateral bulk transporting resistance, that is generally independent on the applied pressure, can be eliminated through simply maximizing the area of conductive electrode (Au film). This method may enhance the dependency of sensor's resistance on the variable contact area caused by the applied pressure, and thus enhance sensor's sensitivity spontaneously.

\subsection{Ultra-fast Response Time and Interpretation}

Another important requirement for high-performance pressure sensor is fast response time [39]. However, the response time of the reported flexible pressure sensors are generally at the level of milliseconds or larger [12,16,18-21], which may limit their applications in the ultra-fast and high frequency detection. The response time of our sensor was tested under ultrafast stimulus. The signals of mechanical vibration, produced by a mixer operating in high speed, can be detected by our sensor and recorded through the triggered mode of oscilloscope (Figure 5a). In order to calculate the response time, we magnified the responsive edge shown as Figure 5b,c. From the sensing curve, we confirmed the response time and relaxation time are about 2 and $74 \mu \mathrm{s}$, respectively, which represent the fastest level so far. To quantitatively understand the improved response characteristics, Nano-indentation was used to characterize elastic properties of pure PDMS and composite PDMS/CNT (Figure 5d). The elastic modulus of pure PDMS films is $13 \mathrm{MPa}$, which is consistent with the previous studies [40,41]. In contrast, the corresponding value of composite PDMS/CNT layer are $23 \mathrm{MPa}$, which is nearly two times larger than that of pure PDMS (Figure 5e). It is primary cause for ultra-fast response of our sensors. These results indicate that the response time of flexible pressure sensor can be shortened through enhancing the elastic modulus of polymer elastomers.
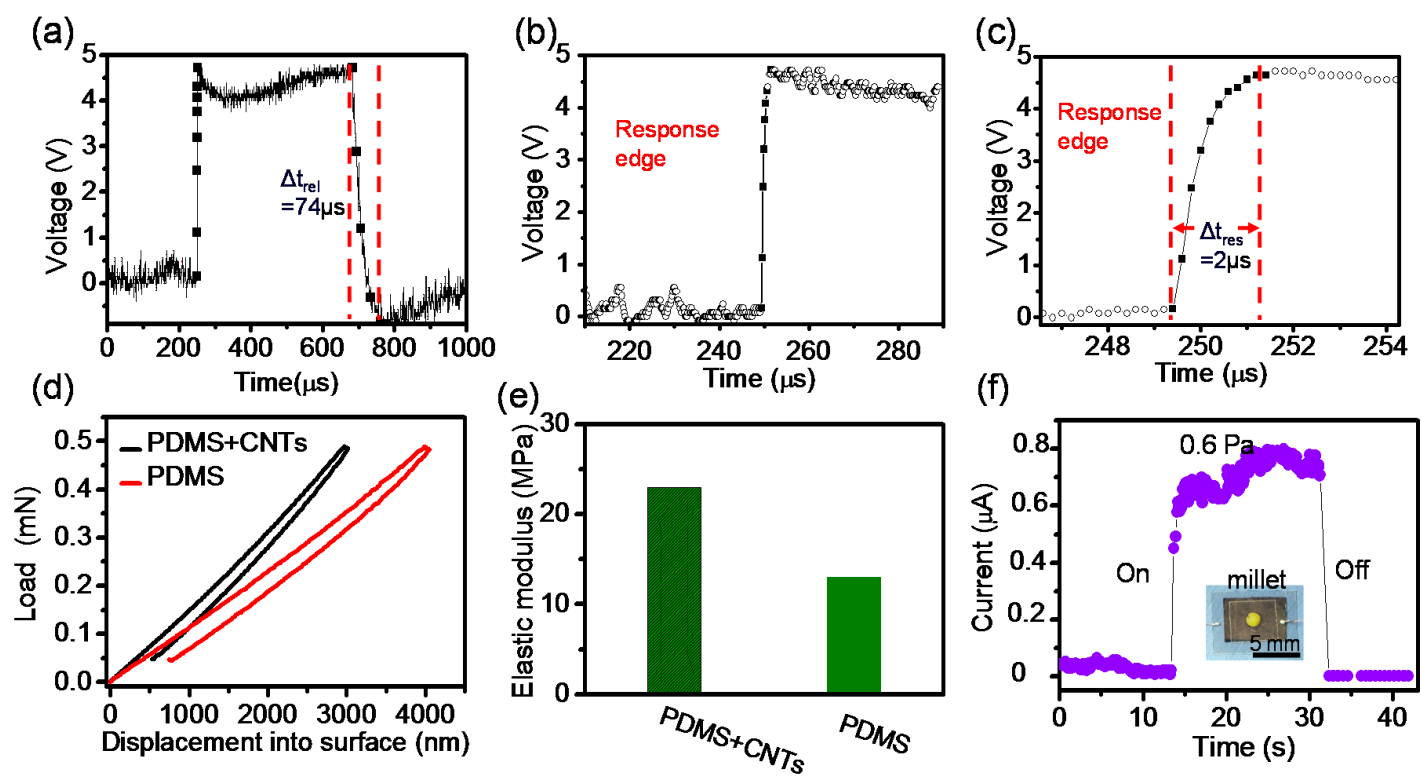

Figure 5. (a) The response of our sensor for a mechanical vibration; (b) Magnified sensor responses extracted from Figure 3a; (c) the further magnified responsive edge, which show the response time is $2 \mu \mathrm{s}$. Nano-indentation was used to characterize pure poly (dimethylsiloxane) (PDMS) and composite PDMS/carbon nanotubes (CNT) films; (d) The different indentation depths of composite films and pure PDMS films under the same load; (e) Elastic modulus of flexible films; (f) The current changes with the application and removal of a millet $(0.6 \mathrm{~Pa})$ under applied voltage of $5 \mathrm{~V}$. 
On the other hand, limit of detection is another important parameter of sensor too. To test it, a millet with a weight of $3 \mathrm{mg}$ corresponding to a tiny pressure of $0.6 \mathrm{~Pa}$ was loaded on and released from the sensor, which resulted in a remarkable change of output current value (Figure $5 f$ ).

\subsection{Typical FunctionalitiesDemonstration}

Our pressure sensors have ultra-fast response time, low detection limit and high sensitivity. They can be used to detect vibrations with various frequencies. Similar to the application of previously reported pressure sensors, the human pulse waveform was monitored firstly (Figure S6). Then the sensors were fixed on different mechanical vibration sources to detect the corresponding vibrations (Figure 6a). Under operation, a mechanical pump can provide a series of vibrations with different frequencies. These vibrations were calibrated by commercial vibration sensor, and the relationship between accelerations and frequencies is shown as Figure $6 \mathrm{~b}$. Our pressure sensors can routinely measure the intense vibrations at 50 and $100 \mathrm{~Hz}$ (Figure 6c, Video S1). Simultaneously, the weak vibrations at 25 and $270 \mathrm{~Hz}$ can also be detected (Figure 6c), indicating the high performance. In fact, our sensor with high sensitivity holds promise for detection higher frequency, which could however be realized due to the lack of reliable high-frequency vibration generator.

(a)

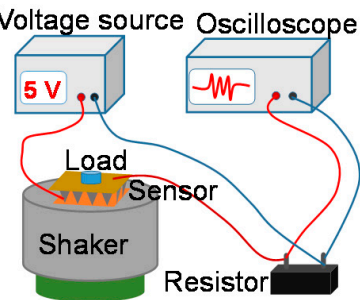

(c)

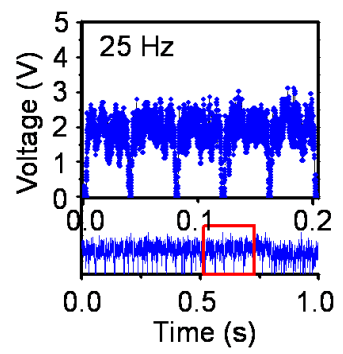

(b)
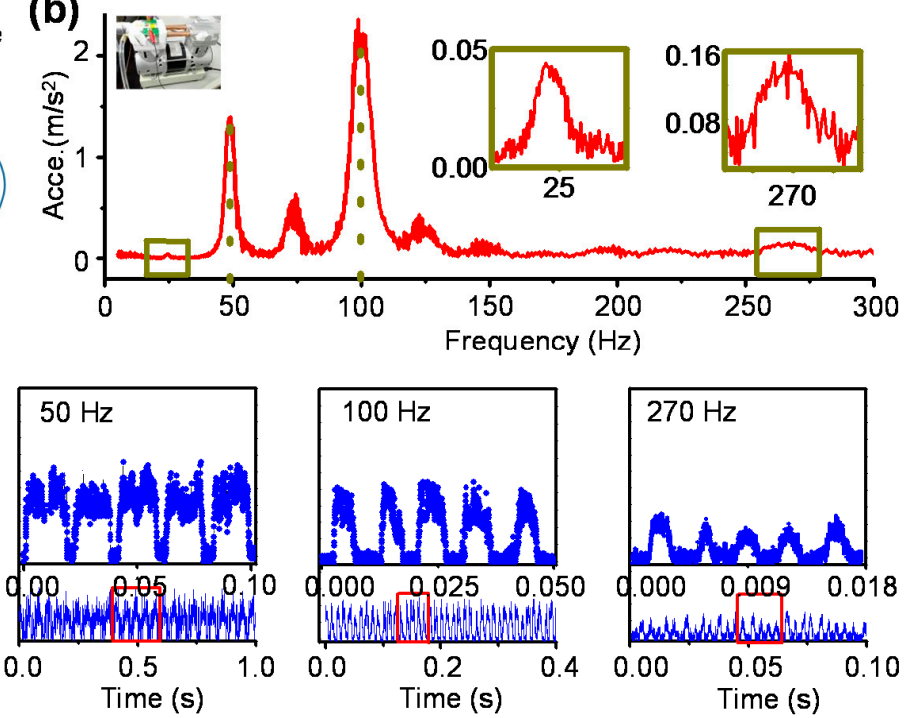

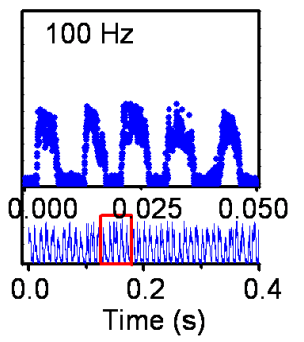

Figure 6. Mechanical vibrations detection. (a) The schematic illustration of mechanical vibration measurement platform. The vibrations provided by mechanical pump; (b) the relationship between accelerations and frequencies, which was calibrated by standard vibration sensor; (c) the typical vibrations with frequencies of 25,50, 100 and $270 \mathrm{~Hz}$ were detected by our sensor.

To test the generality of our sensor for mechanical vibrations detection, the variable-frequency vibrations below hundreds of $\mathrm{Hz}$ provided by mixer under varying operating speeds were monitored by our sensor (Video S2). At same time, the vibrations with the fixed-frequency of $50 \mathrm{~Hz}$ provided by another pump, $25 \mathrm{~Hz}$ (Video S3) and $220 \mathrm{~Hz}$ provided by mixer under different operating speed were detected (Figure S7a-c). In addition, we show the real-time $V$-t curves of 1000 times stimulus based on $50 \mathrm{~Hz}$ vibration (Figure S7d). The sensor maintain good performance after extremely fast multi-times stimulus, which confirmed the good repeatability of the sensor.

On the other hand, our sensors have large pressure measurement rang (0 Pa to $110 \mathrm{kPa}$ ) coupled with the useful sensitivity $\left(14.66 \mathrm{kPa}^{-1}\right)$ in the high pressure region. Meanwhile, the higher elastic modulus of the conductive film may help them to avoid wrinkling under operating in high pressure range. There for they can be used to fabricate flexible air pressure sensors. We propose a packing 
method that seals an amount of gas between two layers of the sensor to complete the fabrication of air pressure sensor schematically illustrated as Figure 7.
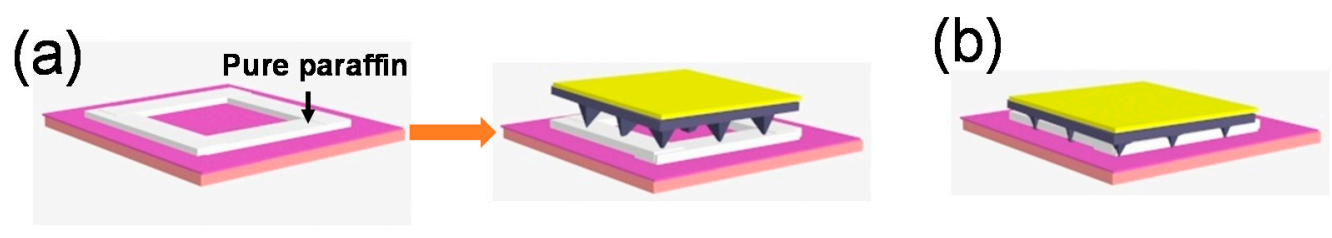

Figure 7. Schematic illustration of the air pressure sensors fabrication, (a) high-purity paraffin was painted on the ITO-PET layer with a closed line frame, and the conductive layer was laminated onto ITO-PET layer; (b) Two layers are sealed through annealing under various vacuum.

To measure the functionality of packaged sensors (PS) for detecting air pressure changes, an elaborate air pressure control and signal record system was built as schematically illustrated in Figure 8a. Real-time air pressures sensing tests of a device packaged at atmosphere were performed under a series of vacuum levels (Figure $8 \mathrm{~b}$, Video S4). $P_{0}$ is the atmospheric pressure $(101 \mathrm{kPa})$ of our laboratory (Suzhou, China). If we turn on the pump, the vacuum levels of vacuum chamber can be changed and hold at random values such as $95,89,80,73,61 \mathrm{kPa}$ defined as $P_{1}, P_{2}, P_{3}, P_{4}, P_{5}$. The reason for choosing these vacuum levels is that they approximately coincide to the atmosphere of Xi'an, Hohhot, Kunming, Geermu, Lhasa (Figure 8b inset), which are Chinese cities. Simultaneously, the responses of PS for the continuous change of air pressure (101 to $60 \mathrm{kPa}$ ) was recorded (Figure 8c). Figure $8 \mathrm{~d}$ demonstrates that the device still obeys Ohm's law even under extremely high pressures. In addition, the air pressure measurement range of PS can be adjusted though tuning the vacuum levels of package stage. For instance, the air pressure measurement range of device packaged at 100 $\mathrm{Pa}$ is 6 to $101 \mathrm{kPa}$ (Figure S8), which is one order of magnitude wider than that of device packaged under atmosphere.
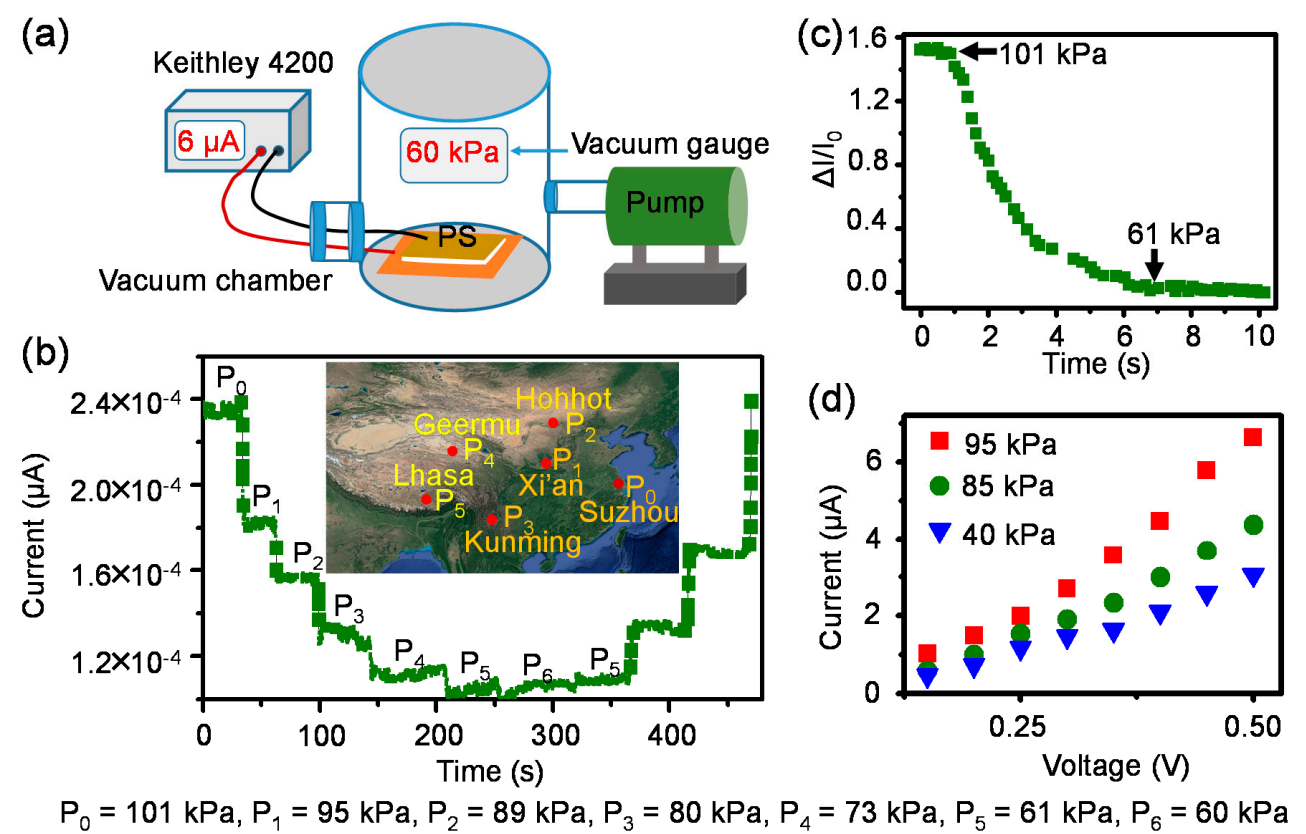

Figure 8. Air pressure detection. (a) The schematic illustration of air pressure control and signal record system. The response of device packed under atmosphere for the air pressure changes; (b) real-time output current under different air pressure levels; (c) Current response under the vacuum level continuously changed from 101 to $60 \mathrm{kPa}$; (d) current-voltage curves under extremely high pressures. 


\section{Conclusions}

We prepared a piezoresistive pressure sensors with sensitivity of $474.13 \mathrm{kPa}^{-1}$ and response time of $2 \mu \mathrm{s}$, which are realized through eliminating the pressure independent lateral bulk transporting resistance and enhancing the elastic modulus of polymer elastomer, respectively. Meanwhile, our sensor have a wide pressure sensing range from $0 \mathrm{~Pa}$ to $110 \mathrm{kPa}$, and low detection limit of $0.6 \mathrm{~Pa}$. Combined these improved performance, we successfully fabricated vibration sensors and air pressure sensors based on our sensors. The vibrations with frequency range from 1 to $\sim 300 \mathrm{~Hz}$ as well as the air pressures from 6 to $101 \mathrm{kPa}$ can be detected accurately.

Supplementary Materials: The following are available online at http:/ / www.mdpi.com/2073-4360/10/6/587/s1.

Author Contributions: Y.M. performed the experiments. H.L., K.W., S.Z. assisted in the device fabrication and characterization. L.L. and Y.M. co-wrote the manuscript. L.L. conceived and supervised the project. All authors discussed the results and commented on the manuscript.

Acknowledgments: The authors are grateful to National Natural Science Foundation of China (21573277, 51503221), National Key Research and Development Program (2016YFB0401100), Key Research Program of Frontier Sciences of Chinese Academy of Sciences (QYZDB-SSW-SLH031), and Natural Foundation of Sciences of Jiangsu Province (BK20150368).

Conflicts of Interest: The authors declare no conflict of interest.

\section{References}

1. Chortos, A.; Liu, J.; Bao, Z. Pursuing prosthetic electronic skin. Nat. Mater. 2016, 15, 937-950. [CrossRef] [PubMed]

2. Lee, S.; Reuveny, A.; Reeder, J.; Lee, S.; Jin, H.; Liu, Q.; Yokota, T.; Sekitani, T.; Isoyama, T.; Abe, Y.; et al. A transparent bending-insensitive pressure sensor. Nat. Nanotechnol. 2016, 11, 472-478. [CrossRef] [PubMed]

3. Shin, S.H.; Ji, S.; Choi, S.; Pyo, K.H.; An, B.W.; Park, J.; Kim, J.; Kim, J.Y.; Lee, K.S.; Kwon, S.Y.; et al. Integrated arrays of air-dielectric graphene transistors as transparent active-matrix pressure sensors for wide pressure ranges. Nat. Commun. 2017, 8, 14950. [CrossRef] [PubMed]

4. Lin, L.; Xie, Y.; Wang, S.; Wu, W.; Niu, S.; Wen, X.; Wang, Z.L. Triboelectric Active Sensor Array for self-powered Static and dynamic pressure detection. ACS Nano 2013, 7, 8266-8274. [CrossRef] [PubMed]

5. Dagdeviren, C.; Su, Y.; Joe, P.; Yona, R.; Liu, Y.; Kim, Y.S.; Huang, Y.; Damadoran, A.R.; Xia, J.; Martin, L.W.; et al. Conformable amplified lead zirconatetitanate sensors with enhanced piezoelectric response for cutaneous pressure monitoring. Nat. Commun. 2014, 5, 4496. [CrossRef] [PubMed]

6. Liu, X.; Long, Y.Z.; Liao, L.; Duan, X.; Fan, Z. Large-Scale Integration of semiconductor nanowires for high-performance flexible electronics. ACS Nano 2012, 6, 1888-1900. [CrossRef] [PubMed]

7. Mannsfeld, S.C.; Tee, B.C.; Stoltenberg, R.M.; Chen, C.V.; Barman, S.; Muir, B.V.; Sokolov, A.N.; Reese, C.; Bao, Z. Highly sensitive flexible pressure sensors with microstructured rubber dielectric layers. Nat. Mater. 2010, 9, 859-864. [CrossRef] [PubMed]

8. Someya, T.; Sekitani, T.; Iba, S.; Kato, Y.; Kawaguchi, H.; Sakurai, T. A large-area, flexiblepressure sensor matrix with organic field-effect transistors for artificial skin applications. Proc. Natl. Acad. Sci. USA 2004, 101, 9966-9970. [CrossRef] [PubMed]

9. Schwartz, G.; Tee, B.C.; Mei, J.; Appleton, A.L.; Kim, D.H.; Wang, H.; Bao, Z. Flexiblepolymer transistors with high pressure sensitivity for application in electronic skin andhealth monitoring. Nat. Commun. 2013, 4, 1859. [CrossRef] [PubMed]

10. Wang, X.W.; Gu, Y.; Xiong, Z.P.; Cui, Z.; Zhang, T. Silk-Molded Flexible, Ultrasensitive, and Highly Stable Electronic Skin for Monitoring Human Physiological Signals. Adv. Mater. 2014, 26, 1336-1342. [CrossRef] [PubMed]

11. Someya, T.; Kato, Y.; Sekitani, T.; Iba, S.; Noguchi, Y.; Murase, Y.; Kawaguchi, H.; Sakurai, T. Conformable, flexible, large-area networks of pressure and thermal sensors with organic transistor active matrixes. Proc. Natl. Acad. Sci. USA 2005, 102, 12321-12325. [CrossRef] [PubMed]

12. Gong, S.; Schwalb, W.; Wang, Y.; Chen, Y.; Tang, Y.; Si, J.; Shirinzadeh, B.; Cheng, W. Awearable and highly sensitive pressure sensor with ultrathin gold nanowires. Nat. Commun. 2014, 5, 3132. [CrossRef] [PubMed] 
13. Kim, G.; Cho, S.; Chang, K.; Kim, W.S.; Kang, H.; Ryu, S.P.; Myoung, J.; Park, J.; Park, C.; Shim, W. Spatially Pressure-Mapped Thermochromic Interactive Senso. Adv. Mater. 2017, 29, 1606120. [CrossRef] [PubMed]

14. Zhao, S.; Zhu, R. Electronic Skin with Multifunction Sensors Based on Thermosensation. Adv. Mater. 2017, 29, 1606151. [CrossRef] [PubMed]

15. Jian, M.; Xia, K.; Wang, Q.; Yin, Z.; Wang, H.; Wang, C.; Xie, H.; Zhang, M.; Zhang, Y. Flexible and Highly Sensitive Pressure Sensors Based on Bionic Hierarchical Structures. Adv. Funct. Mater. 2017, 27, 1606066. [CrossRef]

16. Zhu, B.; Niu, Z.; Wang, H.; Leow, W.R.; Wang, H.; Li, Y.; Zheng, L.; Wei, J.; Huo, F.; Chen, X. Microstructured graphene arrays for highly sensitive flexible tactile sensors. Small 2014, 10, 3625-3631. [CrossRef] [PubMed]

17. Boutry, C.M.; Nguyen, A.; Lawal, Q.O.; Chortos, A.; Rondeau-Gagne, S.; Bao, Z. Sensitive and Biodegradable Pressure Sensor Array for Cardiovascular Monitoring. Adv. Mater. 2015, 27, 6954-6961. [CrossRef] [PubMed]

18. Bae, G.Y.; Pak, S.W.; Kim, D.; Lee, G.; do, H.K.; Chung, Y.; Cho, K. Linearly and Highly Pressure-Sensitive Electronic Skin Based on a Bioinspired Hierarchical Structural Array. Adv. Mater. 2016, 28, 5300-5306. [CrossRef] [PubMed]

19. Wang, Z.; Wang, S.; Zeng, J.; Ren, X.; Chee, A.J.; Yiu, B.Y.; Chung, W.C.; Yang, Y.; Yu, A.C.; Roberts, R.C.; et al. High Sensitivity, Wearable, Piezoresistive Pressure Sensors Based on Irregular Microhump Structures and Its Applications in Body Motion Sensing. Small 2016, 12, 3827-3836. [CrossRef] [PubMed]

20. Kim, K.H.; Hong, S.K.; Jang, N.S.; Ha, S.H.; Lee, H.W.; Kim, J.M. Wearable ResistivePressure Sensor Based on Highly Flexible Carbon Composite Conductors with Irregular Surface Morphology. ACS Appl. Mater. Interfaces 2017, 9, 17499-17507. [CrossRef] [PubMed]

21. Joo, Y.; Yoon, J.; Ha, J.; Kim, T.; Lee, S.; Lee, B.; Pang, C.; Hong, Y. Highly Sensitive and Bendable Capacitive Pressure Sensor and Its Application to $1 \mathrm{~V}$ Operation Pressure-Sensitive Transistor. Adv. Electron. Mater. 2017, 3, 1600455. [CrossRef]

22. Zhang, F.; Zang, Y.; Huang, D.; Di, C.A.; Zhu, D. Flexible and self-powered temperature-pressure dual-parameter sensors using microstructure-frame-supported organic thermoelectric materials. Nat. Commun. 2015, 6, 8356. [CrossRef] [PubMed]

23. Zang, Y.; Zhang, F.; Huang, D.; Gao, X.; Di, C.A.; Zhu, D. Flexible suspended gate organicthin-film transistors for ultra-sensitive pressure detection. Nat. Commun. 2015, 6, 6269. [CrossRef] [PubMed]

24. Zhao, X.; Hua, Q.; Yu, R.; Zhang, Y.; Pan, C. Stretchable and Wearable Multifunctional Sensor Array as Artificial Electronic Skin for Static and Dynamic Strain Mapping. Adv. Electron. Mater. 2015, 1, 1500142. [CrossRef]

25. Choong, C.L.; Shim, M.B.; Lee, B.S.; Jeon, S.; Ko, D.S.; Kang, T.H.; Bae, J.; Lee, S.H.; Byun, K.E.; Im, J.; et al. Highly Stretchable Resistive Pressure Sensors Usinga Conductive Elastomeric Composite on a Micropyramid Array. Adv. Mater. 2014, 26, 3451-3458. [CrossRef] [PubMed]

26. Luo, N.; Dai, W.; Li, C.; Zhou, Z.; Lu, L.; Poon, C.C.Y.; Chen, S.-C.; Zhang, Y.; Zhao, N. Flexible Piezoresistive Sensor Patch Enabling Ultralow Power Cuffless Blood Pressure Measurement. Adv. Funct. Mater. 2016, 26, 1178-1187. [CrossRef]

27. Chen, Z.; Wang, Z.; Li, X.; Lin, Y.; Luo, N.; Long, M.; Zhao, N.; Xu, J.B. FlexiblePiezoelectric-Induced Pressure Sensors for Static Measurements Based on Nanowires/Graphene Heterostructures. ACS Nano 2017, 11, 4507-4513. [CrossRef] [PubMed]

28. Chun, K.Y.; Son, Y.J.; Han, C.S. Highly Sensitive and Patchable Pressure Sensors Mimicking Ion-Channel-Engaged Sensory Organs. ACS Nano 2016, 10, 4550-4558. [CrossRef] [PubMed]

29. Tee, B.C.; Wang, C.; Allen, R.; Bao, Z. An electrically and mechanically self-healing composite with pressureand flexion-sensitive properties for electronic skin applications. Nat. Nanotechnol. 2012, 7, 825-832. [CrossRef] [PubMed]

30. Lou, Z.; Chen, S.; Wang, L.; Jiang, K.; Shen, G. An ultra-sensitive and rapid response speed graphene pressure sensors for electronic skin and health monitoring. Nano Energy 2016, 23, 7-14. [CrossRef]

31. Shin, K.-Y.; Lee, J.S.; Jang, J. Highly sensitive, wearable and wireless pressure sensor using free-standing $\mathrm{ZnO}$ nanoneedle/PVDF hybrid thin film for heart rate monitoring. Nano Energy 2016, 22, 95-104. [CrossRef]

32. Xu, B.; Wang, C.; Wang, D.N.; Liu, Y.; Li, Y. Fiber-tip gas pressure sensor based on dual capillaries. Opt. Express 2015, 23, 23484-23492. [CrossRef] [PubMed] 
33. Li, Z.; Liao, C.; Wang, Y.; Xu, L.; Wang, D.; Dong, X.; Liu, S.; Wang, Q.; Yang, K.; Zhou, J. Highly-sensitive gas pressure sensor using twin-core fiber based in-line Mach-Zehnder interferometer. Opt. Express 2015, 23, 6673-6678. [CrossRef] [PubMed]

34. Dong, R.G.; Welcome, D.E.; Wu, J.Z. Frequency weightings based on biodynamics of fingers-hand-arm system. Ind. Health 2005, 43, 516-526. [CrossRef] [PubMed]

35. Zhu, Y.F.; Ma, C.; Zhang, W.; Zhang, R.P.; Koratkar, N.; Liang, J. Alignment of multiwalled carbon nanotubes in bulk epoxy composites via electric field. J. Appl. Phys. 2009, 105, 054319. [CrossRef]

36. Sun, G.; Zhang, X.; Lin, R.; Yang, J.; Zhang, H.; Chen, P. Hybrid fibers made of molybdenum disulfide, reduced graphene oxide, and multi-walled carbon nanotubes for solid-state, flexible, asymmetric supercapacitors. Angew. Chem. Int. Ed. 2015, 54, 4651-4656. [CrossRef] [PubMed]

37. Holm, R. Electric Contacts: Theory and Application; Springer: New York, NY, USA, 1967.

38. Zhang, Z. The electrostatic energy and potential produced by a uniformly charged prolate ellipsoid of revolution. Coll. Phys. 1994, 13, 12.

39. Shao, Q.; Niu, Z.Q.; Hirtz, M.; Jiang, L.; Liu, Y.J.; Wang, Z.H.; Chen, X.D. High-Performance and Tailorable Pressure Sensor Based on Ultrathin Conductive PolymerFilm. Small 2014, 10, 1466-1472. [CrossRef] [PubMed]

40. Gupta, S.; Carrillo, F.; Li, C.; Pruitt, L.; Puttlitz, C. Adhesive forces significantly affectelastic modulus determination of soft polymeric materials in nanoindentation. Mater. Lett. 2007, 61, 448-451. [CrossRef]

41. Carrillo, F.; Gupta, S.; Balooch, M.; Marshall, S.J.; Marshall, G.W.; Pruitt, L.; Puttlitz, C.M. Nanoindentation of polydimethylsiloxane elastomers: Effect of crosslinking, work of adhesion, and fluid environment on elastic modulus. J. Mater. Res. 2005, 20, 2820-2830. [CrossRef]

(C) 2018 by the authors. Licensee MDPI, Basel, Switzerland. This article is an open access article distributed under the terms and conditions of the Creative Commons Attribution (CC BY) license (http:/ / creativecommons.org/licenses/by/4.0/). 